\title{
Avaliação da atividade antileishmania in vitro de Tanacetum vulgare (Asteraceae)
}

\author{
Bruno Bezerra Jensen ${ }^{a b *}$, Claudia Dantas Comandolli-Wyrepkowski ${ }^{b}$, Angela Maria Comapa Barros ${ }^{\mathrm{b}}$, Fabiane \\ Veloso Soares ${ }^{\mathrm{ab}}$, Iryna Grafovac, Andriy Grafovc, Antonia Maria Ramos Franco ${ }^{\mathrm{a}}$ \\ ${ }^{a}$ Laboratório de Leishmaniose e Doenças de Chagas, Instituto Nacional de Pesquisas da Amazônia, Manaus, 69067-375, \\ Brasil. *brunobjensenfarma@gmail.com \\ ${ }^{b}$ Programa de Pós-graduação Multi-Institucional em Biotecnologia, Universidade Federal do Amazonas, Manaus, 69067-005, Brasil. \\ ${ }^{c}$ Departamento de Química, Universidade de Helsinki, Helsinki, Finlândia.
}

Recebido: 14 janeiro 2017 / Aceito: 17 março 2017 / Publicado online: 23 maio 2017

\begin{abstract}
Resumo
A Leishmaniose é considerada uma doença negligenciada. Nos últimos anos estudos tem demonstrado que várias plantas possuem atividade contra esses patógenos, servindo de base para a formulação de fitoterápicos ou como alvo para a obtenção de princípios ativos contra a doença. Esta pesquisa teve como objetivo avaliar a atividade biológica in vitro antileishmania dos extratos hexânicos e metanólicos das flores e folhas de Tanacetum vulgare contra Leishmania amazonensis e Leishmania guyanensis. Foi realizada a extração das flores e folhas do material vegetal a partir dos solventes hexânico e metanólico. A atividade antileishmania dos extratos de $T$. vulgare foram avaliadas de acordo com a inibição do crescimento e a mortalidade de promastigotas de L. amazonensis e L. guyanensis, nos períodos de 24,48 e 72 h após incubação a $25^{\circ} \mathrm{C}$ na presença dos extratos com concentrações de 31,25 a $500 \mu \mathrm{g} \mathrm{mL} \mathrm{mL}^{-1}$. Os resultados mostraram que o extrato metanólico da flor apresentou atividade contra L. amazonensis ( $\mathrm{IC}_{50}=31,5 \mu \mathrm{g} \mathrm{mL}^{-1}$ ) e L. guyanensis $\left(\mathrm{IC}_{50}<100 \mu \mathrm{g} \mathrm{mL} \mathrm{L}^{-1}\right.$ ), em $72 \mathrm{~h}$ de incubação. Portanto, verifica-se que o extrato metanólico da flor de $T$. vulgare desempenhou melhor resultado quanto à atividade antileishmania, permitindo a possibilidade de estudos futuros para determinar a sua eficácia terapêutica.
\end{abstract}

Palavras-chave: bioensaios; Leishmaniose tegumentar; Leishmania amazonensis; Leishmania guyanensis

\section{Evaluation of activity anti-leishmania in vitro of Tanacetum vulgare (Asteraceae)}

\begin{abstract}
Leishmaniasis is considered a neglected disease. In recent years studies have shown that several plants have activity against these pathogens, serving as the basis for the formulation of herbal medicines or as a target for obtaining active principles against the disease. This study aimed to evaluate the in vitro antileishmania biological activity of the hexanic and methanolic extracts of the flowers and leaves of Tanacetum vulgare against Leishmania amazonensis and L. guyanensis. The flowers and leaves of the plant material were extracted from the hexane and methanolic solvents. The antileishmania activity of T. vulgare extracts was evaluated according to the inhibition of growth and mortality of L. amazonensis and L. guyanensis promastigotes at periods of 24,48 and $72 \mathrm{~h}$ after incubation at $25^{\circ} \mathrm{C}$ in the presence of extracts with concentrations of 31.25 to $500 \mu \mathrm{g} \mathrm{mL}^{-1}$. The results showed that the extract metanolic of the flower showed activity against L. amazonensis (IC50 $=31.5 \mu \mathrm{g} \mathrm{mL} \mathrm{m}^{-1}$ ) and L. guyanensis (IC50 < $100 \mu \mathrm{g} \mathrm{mL}^{-1}$ ) in $72 \mathrm{~h}$ of incubation. Therefore, it is verified that the methanolic extract of the T. vulgare flower performed better in relation to antileishmania activity, allowing the possibility of future studies to determine its therapeutic efficacy.
\end{abstract}

Key-words: bioassays; cutaneous leishmaniasis; Leishmania amazonensis; Leishmania guyanensis

\section{Introdução}

A Leishmaniose Tegumentar Americana (LTA) é uma doença tropical negligenciada de grande significância para saúde pública (Pigott et al., 2014). Sua transmissão ocorre de forma vetorial através da fêmea de flebotomíneos do gênero Lutzomyia (WHO, 2010; Dostalova e Volf, 2012; Brasil, 2017). A doença pode apresentar diferentes formas clínicas dependendo da espécie de Leishmania envolvida e da relação do parasito com seu hospedeiro (Mota e Miranda, 2011).

A Leishmaniose cutânea é considerada endêmica em 98 países, com registro anual de 220.000 casos, distribuídos nos cinco continentes, com números majoritários na América, seguido da Ásia, África, Europa e Oceania (Alvar et al., 2012; Pigott et al., 2014). O Brasil apresentou 237.145 casos, no período de 2005 a 2015, sendo o país da América com maior 
número de registros, com destaque para a região Norte, Centro-Oeste e Nordeste (Alvar et al., 2012; SINAN/SVS/MS, 2017). No Estado do Amazonas a Leishmaniose ocupa o segundo lugar com 2.235 casos, depois da malária com 82.910 , entre as infecções por protozoários que acometem os seres humanos (FVS-AM, 2012). Estes valores estão inerentes à abertura de novas estradas e a instalação de novos núcleos residenciais em áreas onde, previamente, existia densa floresta tropical (Pinheiro et al., 2008).

$\mathrm{O}$ antimonial pentavalente $\left(\mathrm{Sb}^{5+}\right)$ é o medicamento preconizado pelo Ministério da Saúde para combater a Leishmaniose, como primeira escolha, pentamidina e anfontericina B como segunda opção (Brasil, 2017). No entanto, esse esquema terapêutico tem algumas problemáticas, pois tem administração parenteral, exigindo a participação do enfermo e muitos abandonam o tratamento e; possui eficácia limitada e inúmeras reações adversas, podendo causar aborto, insuficiência renal aguda e hipotensão (Silva-Lopez, 2010; Ferreira et al., 2012; Brasil, 2017).

A necessidade de tratamentos com maior eficácia e segurança vem estimulando pesquisas com produtos naturais de plantas com atividade antileishmania, uma vez que os vegetais possuem uma diversidade química de metabólitos secundários estruturalmente únicos.

Tanacetum vulgare L., popularmente conhecido como Catinga de mulata, pertence à família Asteraceae, é uma planta nativa de terrenos úmidos da Europa e Ásia, cultivada como planta ornamental no Brasil. Na região norte, no Amazonas, é cultivada na Reserva de Desenvolvimento Sustentável do Tupé, Manaus-AM, com finalidade medicinal para dor de barriga e como vermífugo, com base no conhecimento popular (Souza, 2010; Guerreiro et al., 2016).

Estudos químicos e farmacológicos de extratos obtidos de T. vulgare tem demonstrado seu potencial biológico antiinflamatório (Schinella et al., 1998; William, 1999), antibacteriano e antifúngico (Pessini et al., 2003), antimalárico (Jansen, 2006), antiviral (Álvarez et al., 2011), com atividade diurética (Lahlou et al., 2007) e também demonstrou ótimos valores de inibição do crescimento in vitro para L. amazonensis e Trypanosoma cruzi (Luize et al., 2005).

Esta pequisa teve por objetivo avaliar a atividade biológica in vitro antileishmania dos extratos hexânicos e metanólicos das flores e folhas de $T$. vulgare (Asteraceae) em $L$. amazonensis e L. guyanensis.

\section{Material e Métodos}

\section{Material vegetal}

Folhas e flores de $T$. vulgare foram coletadas no Parque Savela, na cidade de Helsinki, Finlândia. O material vegetal foi identificado pelo Dr. Henry Vare, e as exsicatas foram depositadas no Herbário do Primeiro Museu de História Natural, sob o número de identificação 197666.

Preparação dos extratos Hexânico e Metanólico das folhas e flores de T. vulgare

O material vegetal foi seco em estufa a temperaturas inferiores a $42{ }^{\circ} \mathrm{C}$. As folhas e flores foram separadas e trituradas em moinho. Os extratos foram obtidos segundo
Cechinel e Yunes (2001), utilizando $250 \mathrm{~mL}$ de hexano (HEX) para cada $100 \mathrm{~g}$ de material pulverizado na primeira etapa da extração e $250 \mathrm{~mL}$ metanol $(\mathrm{MeOH})$ na segunda etapa, ambas em banho de ultrassom, por 20 minutos cada. Foram realizadas três extrações para cada solvente. Após cada banho em ultrassom os extratos foram filtrados e concentrados em evaporador rotatório.

\section{Manutenção de Leishmania}

Foram utilizadas as espécies Leishmania (Leishmania) amazonensis (MHO/BR/2009/IM5584) e Leishmania (Viannia) guyanensis (MHO/BR/95/IM4147), mantidas no Laboratório de Leishmaniose e Doença de Chagas, Coordenação de Sociedade, Ambiente e Saúde, INPA. As formas promastigotas de L. amazonensis e L. guyanensis para utilização nos bioensaios in vitro foram mantidas e cultivadas em meio RPMI 1640 (Himedia) completo, suplementado com $10 \%$ de soro fetal bovino inativado (SFBi) em estufa a $25^{\circ} \mathrm{C}$, de acordo com Jaffe et al. (1984). Foram realizadas curvas de crescimento parasitário de ambas as espécies previamente à realização dos bioensaios.

Determinação da atividade antileishmania em promastigotas e concentração efetiva $\left(I_{50}\right)$ de extratos de T. vulgare

A atividade antileishmania dos extratos de $T$. vulgare foi avaliada pela inibição do crescimento e mortalidade de $L$. amazonensis e L. guyanensis. Para a realização dos bioensaios, as formas promastigotas na fase logarítimica tardia foram centrifugadas, lavadas em salina estéril, diluídas e quantificadas em câmara de Neubauer, sendo ajustadas para obter a concentração parasitária de $2 \times 10^{6}$ células $/ \mathrm{mL}$, de acordo com Fumarola et al. (2004).

Os extratos de $T$. vulgare foram diluídos em dimetilsulfóxido (DMSO - Vetec ${ }^{\circledR} ; 3 \%$ v/v) e em meio RPMI sem adição de SFBi e filtrados em membrane Millipore ${ }^{\circledR}$ 0,22 $\mu \mathrm{m}$ em ambiente estéril. Os extratos foram avaliados nas concentrações de 31,25 a $500 \mu \mathrm{g} \mathrm{mL}^{-1}$. O controle negativo foi o DMSO $(3 \% \mathrm{v} / \mathrm{v})$ e o controle positivo foi Pentacarinat ${ }^{\circledR}$ (Isotionato de pentamidina), na concentração máxima de 10 $\mu \mathrm{g} \mathrm{mL} \mathrm{m}^{-1}$ e mínima de $0,0625 \mu \mathrm{g} \mathrm{mL}^{-1}$. As atividades dos extratos foram avaliadas por contagem de células viáveis após a adição dos extratos em diferentes concentrações na presença de $2 \times 10^{6}$ promastigotas $/ \mathrm{mL}$ e após incubação nos períodos de 24,48 e $72 \mathrm{~h}$, em estufa a $25^{\circ} \mathrm{C}$.

Os bioensaios foram realizados em triplicatas e repetidos duas vezes. A contagem dos parasitas foi realizada em hemocitômetro. A média do número de células vivas foi utilizada para análise da regressão linear e determinação dos valores da $\mathrm{IC}_{50}$ (concentração inibitória para $50 \%$ dos parasitas). Este valor é um importante parâmetro de referência para determinar as amostras mais promissoras, isto é, as que possuírem o menor $\mathrm{IC}_{50}$.

\section{Análise Estatística}

A multiplicação das células parasitárias foi determinada pelas curvas sigmoidais, utilizando-se o software GraphPad Prism 6.0, analisando-se os respectivos intervalos de confiança $95 \%$ e coeficientes lineares. Para a análise estatística foi utilizado o teste de variância (ANOVA). O valor da $\mathrm{IC}_{50}$ foi obtido por análise da regressão linear. 


\section{Resultados e Discussão}

$\mathrm{O}$ extrato $\mathrm{MeOH}$ da flor inibiu mais significativamente o crescimento de L. amazonensis, do que o extrato Hex da flor, principalmente, nas concentrações de 125 a $500 \mu \mathrm{g} \mathrm{mL}^{-1}$, estes nos quais tão eficientes, no tempo de 72 horas em relação ao controle positivo, com Pentacarinat ${ }^{\circledR}$ (Figura 1).

As concentrações 250 e $500 \mu \mathrm{g} \mathrm{mL}^{-1}$ do extrato $\mathrm{MeOH}$ das folhas interferiram no crescimento de promastigotas de $L$. amazonensis, mais do que as concentrações do extrato Hex das folhas além disso, a partir de 72 horas, apresentou eficácia semelhante ao Pentacarinat ${ }^{\circledR}$ (Figura 2).

Somente a concentração de $500 \mu \mathrm{g} \mathrm{mL} \mathrm{L}^{-1}$ do extrato $\mathrm{MeOH}$ da flor apresentou atividade inibitória contra $L$. guyanensis (Figura 3). Todas as concentrações dos extratos $\mathrm{MeOH}$ e Hex da folha de $T$. vulgare, não apresentaram atividade inibitória contra essa espécie (Figura 4).
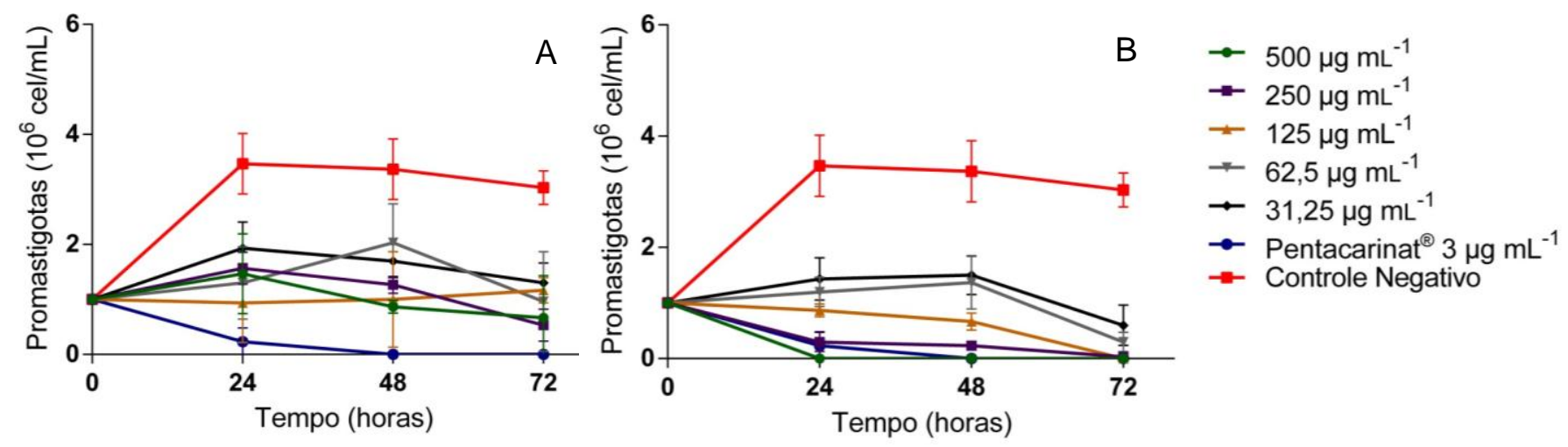

Figura 1. Atividade em 24, 48 e 72 horas dos extratos hexânico (A) e metanólico (B) da flor de Tanacetum vulgare testados em ensaio biológico in vitro contra Leishmania amazonensis.
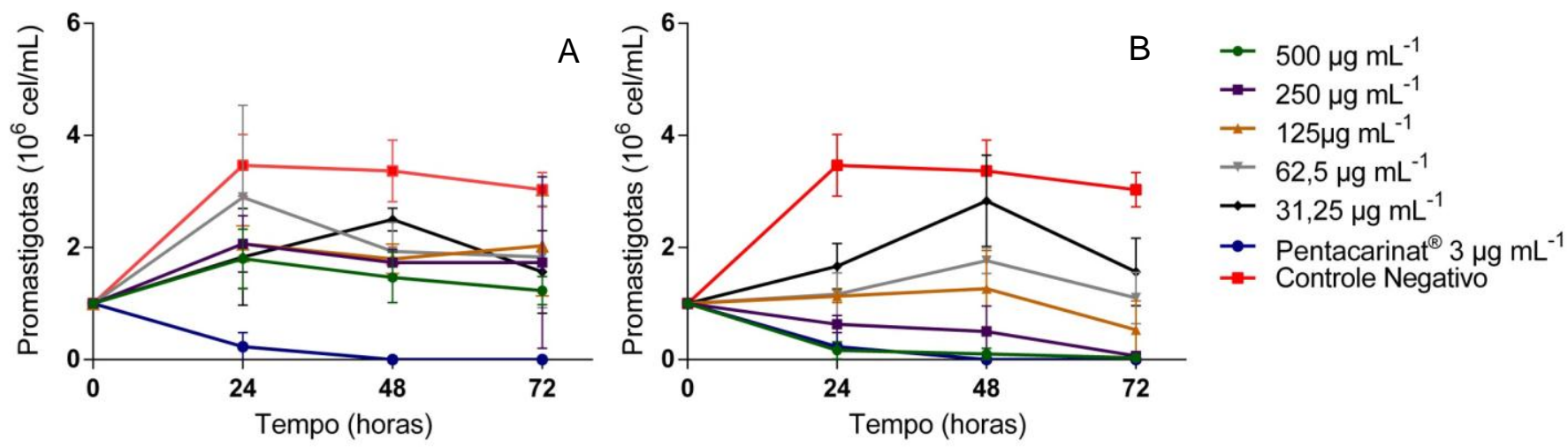

Figura 2. Atividade em 24, 48 e 72 horas dos extratos hexânico (A) e metanólico (B) das folhas de Tanacetum vulgare testados em ensaio biológico in vitro contra Leishmania amazonensis.

Os valores de $\mathrm{IC}_{50}$ encontrados para os extratos $\mathrm{MeOH}$ e Hex de flores e folhas variaram de $31,5 \mathrm{a}>100 \mu \mathrm{g} \mathrm{mL}$ (Tabela 1). Segundo Osorio et al. (2007), as concentrações ativas em ensaios biológicos in vitro, podem ser classificadas como: concentrações altamente ativas $\left(\mathrm{IC}_{50}<10 \mu \mathrm{g} \mathrm{mL} \mathrm{L}^{-1}\right)$, ativas $\left(\mathrm{IC}_{50}\right.$ entre $\left.10-50 \mu \mathrm{g} \mathrm{mL}^{-1}\right)$, moderadamente ativas $\left(\mathrm{IC}_{50}\right.$

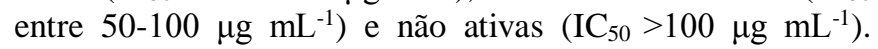
Supracitado, corroborou para análise dos resultados do ensaio biológico com $T$. vulgare, onde os extratos $\mathrm{Hex}$ e $\mathrm{MeOH}$ da flor testados contra $L$. amazonensis, desempenharam $\mathrm{IC}_{50}$ igual a 31,5 $\mu \mathrm{g} \mathrm{mL}^{-1}$, considerados ativos. Luize et al. (2005), observaram que o valor de $\mathrm{IC}_{50}$ do extrato hidroalcólico das folhas de $T$. vulgare contra o parasita $L$. amazonensis foi de
96,4 $\mu \mathrm{g} \mathrm{mL}^{-1}$, moderadamente ativo, resultado semelhante aos extratos $\mathrm{Hex}$ e $\mathrm{MeOH}$ de folhas do presente estudo.

A família Asteraceae está sendo investigada por muitos laboratórios e apresenta importantes perspectivas para o desenvolvimento de medicamentos contra diferentes agentes parasitários, como, Leishmania amazonensis e Trypanosoma cruzi (Schinor et al., 2007), Leishmania donovani e Plasmodium falciparum (Silva-Filho, et al., 2009) e Schistosoma mansoni (Almeida et al., 2016).

Os valores de $\mathrm{IC}_{50}$ encontrados para os extratos $\mathrm{MeOH}$ e Hex de flores e folhas variaram de $31,5 \mathrm{a}>100 \mu \mathrm{g} \mathrm{m}^{-1}$ (Tabela 1). 

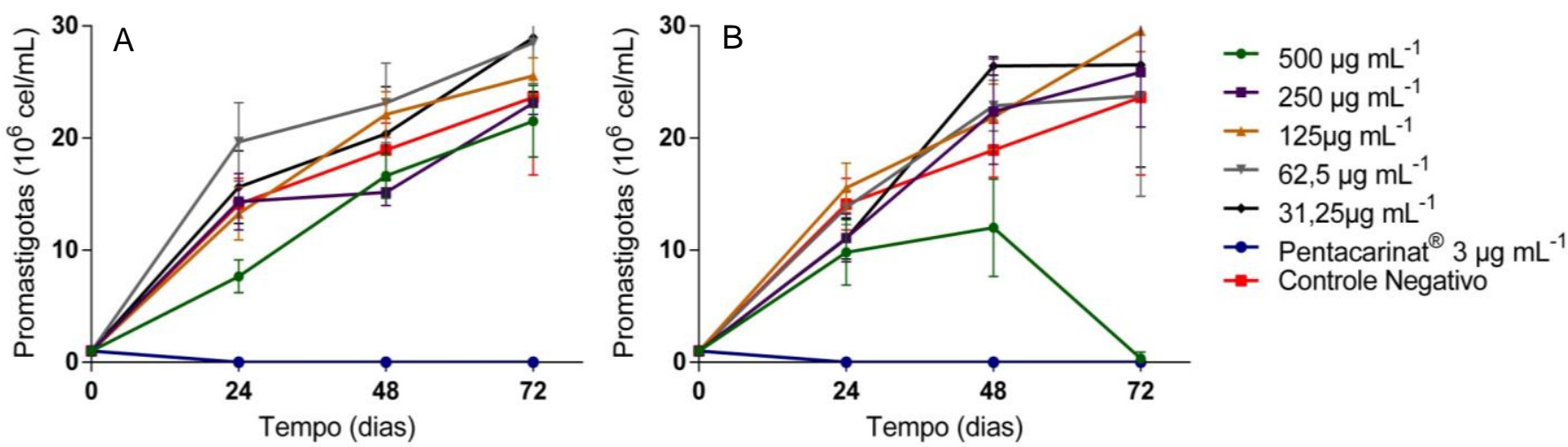

Figura 3. Atividade em 24, 48 e 72 horas dos extratos hexânico (A) e metanólico (B) da flor de Tanacetum vulgare testados em ensaio biológico in vitro contra Leishmania guyanensis.
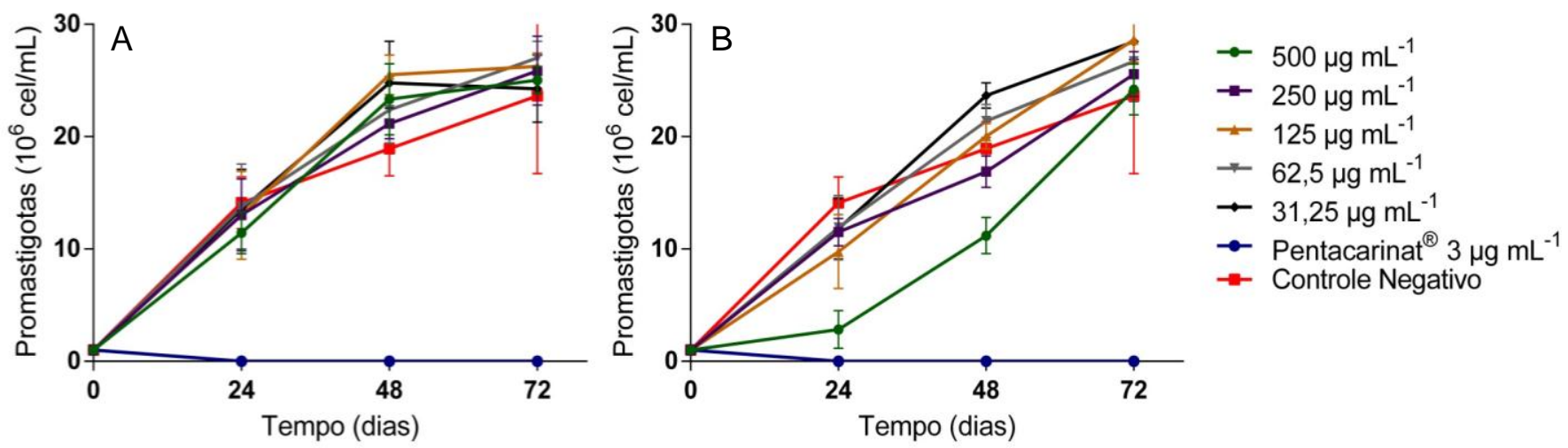

Figura 4. Atividade em 24, 48 e 72 horas dos extratos hexânico (A) e metanólico (B) das folhas de Tanacetum vulgare testados em ensaio biológico in vitro contra Leishmania guyanensis.

Segundo Osorio et al. (2007), as concentrações ativas em ensaios biológicos in vitro, podem ser classificadas como: concentrações altamente ativas $\left(\mathrm{IC}_{50}<10 \mu \mathrm{g} \mathrm{mL}^{-1}\right.$ ), ativas $\left(\mathrm{IC}_{50}\right.$ entre $\left.10-50 \mu \mathrm{g} \mathrm{mL}^{-1}\right)$, moderadamente ativas $\left(\mathrm{IC}_{50}\right.$ entre $\left.50-100 \mu \mathrm{g} \mathrm{mL}^{-1}\right)$ e não ativas $\left(\mathrm{IC}_{50}>100 \mu \mathrm{g} \mathrm{mL}^{-1}\right)$.

Tabela 1 - Valores de $\mathrm{IC}_{50}$ antileishmania contra promastigotas dos extratos obtidos das flores e folhas de T. vulgare.

\begin{tabular}{lcc}
\hline \multirow{2}{*}{ Amostra } & \multicolumn{2}{c}{$\mathrm{IC}_{50}\left(\mu \mathrm{g} \mathrm{mL}^{-1}\right)$} \\
\cline { 2 - 3 } & $\begin{array}{c}\text { L. amazonensis } \\
\text { (IM 5584) }\end{array}$ & $\begin{array}{c}\text { L. } \text { guyanensis } \\
\text { (IM 4147) }\end{array}$ \\
\cline { 2 - 3 } & $72 \mathrm{~h}$ & $72 \mathrm{~h}$ \\
\hline Hex Flor & 31,5 & $>100$ \\
Hex Folha & $>100$ & $>100$ \\
MeOH Flor & 31,5 & 94,1 \\
MeOH Folha & 60,2 & $>100$ \\
Pentacarinat $^{\circledR}$ & 1,6 & 1,3 \\
\hline
\end{tabular}

Supracitado, corroborou para análise dos resultados do ensaio biológico com $T$. vulgare, onde os extratos Hex e $\mathrm{MeOH}$ da flor testados contra $L$. amazonensis,

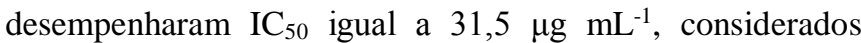
ativos. Luize et al. (2005), observaram que o valor de $\mathrm{IC}_{50}$ do extrato hidroalcólico das folhas de T. vulgare contra o parasita L. amazonensis foi de $96,4 \mu \mathrm{g} \mathrm{mL}{ }^{-1}$, moderadamente ativo, resultado semelhante aos extratos $\mathrm{Hex}$ e $\mathrm{MeOH}$ de folhas do presente estudo.

A família Asteraceae está sendo investigada por muitos laboratórios e apresenta importantes perspectivas para o desenvolvimento de medicamentos contra diferentes agentes parasitários, como, Leishmania amazonensis e Trypanosoma cruzi (Schinor et al., 2007), Leishmania donovani e Plasmodium falciparum (Silva-Filho, et al., 2009) e Schistosoma mansoni (Almeida et al., 2016).

Entretanto, existe outra espécie vegetal com atividade antileishmania, também pertencente da família Asteraceae, onde o isolado de $T$. parthenium, guaianolida apresentou a $\mathrm{IC}_{50}$ igual a $2,6 \mu \mathrm{g} \mathrm{mL}^{-1}$, com valor de alta atividade contra o crescimento de Leishmania (Silva et al., 2010). Além disso, tem sido demonstrado que a fração diclorometano dessa planta apresentou a $\mathrm{IC}_{50}$ igual a $2,4 \mu \mathrm{g} \mathrm{mL}^{-1}$ para promastigota de $L$. amazonensis, inclusive com resultado efetivo avaliado em camundongos infectados com a mesma espécie do parasita (Rabito et al., 2014).

Para o ensaio com L. guyanensis, o extrato $\mathrm{MeOH}$ de flor apresentou atividade moderada com $\mathrm{IC}_{50}<$ que $100 \mu \mathrm{gL}^{-1}$. Os demais extratos não apresentaram atividade contra 0 parasita e com as $\mathrm{IC}_{50}$ correspondente a $>100 \mu \mathrm{g} \mathrm{mL}^{-1}$ no 
tempo de $72 \mathrm{~h}$ (Tabela 1).

Segundo Bednarczuk et al. (2010), a estratégia para novos medicamentos para o tratamento de qualquer doença, deverá passar por ensaios biológicos in vitro com a finalidade de realizar uma triagem de substâncias inerentes de plantas ou sintéticas para avaliar efeitos citotóxicos, para assegurar em um ensaio in vivo.

Constatou-se que o extrato $\mathrm{MeOH}$ de flor possui atividade antileishmania contra L. amazonensis e L. guyanensis devendo ser melhor estudado em ensaios posteriores, já que o $T$. vulgare é alvo de diferentes estudos com atividades farmacológicas definidas, como ação antiinflamatória (William, 1999), antimalárica (Jansen, 2006) e anti-helmíntico (Goldinho et al., 2014), indicando-se promissora a realização de estudos voltados para avaliação quanto a sua eficácia terapêutica.

\section{Conclusão}

$\mathrm{O}$ extrato $\mathrm{MeOH}$ da flor de T. vulgare apresentou resultado expressivo contra o crescimento de $L$. amazonensis e $L$. guyanensis, com valores reduzidos da $\mathrm{IC}_{50}$. Os resultados obtidos reportam a necessidade de estudos mais apurados que visem determinar os compostos químicos presentes nos extratos para que futuramente possam ser avaliados em outros testes in vitro, parasito na forma amastigota e citotoxicidade.

\section{Referências}

Almeida, L.M.S.; Carvalho, L.S.A.; Gazolla, M.C.; Pinto, P.L.S.; Silva, M.P.N.; Moraes, J.; Filho, A.A.S. 2016. Flavanoids and sesquiterpene lactones from Artemisia absinthium na Tanacetum parthenium against Schistosoma mansoni worms. Evidence-Based Complementary and Alternative Medicine, 2016(ID 9521349): 1-10.

Alvar, J.; Vélez, I.D.; Bern, C.; Herrero, M.; Desjeux, P.; Cano, J.; Jannin, J.; DenBoer, M. 2012. WHO Leishmaniasis Control Team. Leishmaniasis Worldwide and global estimates of its incidence. PLoSOne, 7(5): e35671.

Álvarez, A.L.; Habtmariam, S.; Juan-Badaturuge, M.; Jackson, C.; Parra, F. 2011. In vitro Anti HSV-1 and HSV-2 Activy of Tanacetum vulgare Extracts and Isolated Compounds: An Approach to their Mechanisms of Action. Phytotherapy Research.

Brasil. 2017. Manual de Vigilância da Leishmaniose Tegumentar Americana. Ministério da Saúde, Secretaria de Vigilância em Saúde. 2. ed. Brasília: Editora do Ministério da Saúde. 190p.

Bednarczuk, V.O.; Verdam, M.C.S.; Miguel, O.G. 2010. Testes in vitro e in vivo utilizados na triagem toxicológica de produtos naturais. Visão Acâdemica, 11(2): 1518-5192.

Cechinel, V.; Yunes, R.A. 2001. Estudo químico de plantas medicinais orientado para análise biológica. Obtenção, determinação e modificação estrutural de compostos bioativos. Chapecó: Argos Editora Universitária. 523p.

Dostalova, A.; Volf, P. 2012. Leishmania development in sand flies: parasitevector interactions overview. Parasites \& Vectors, (5): 276.

Ferreira, C.C.; Marochio, G.G.; Partata, A.K. 2012. Estudo sobre a Leishmaniose Tegumentar Americana com Enfoque na Farmacoterapia. Revista Científica do ITPAC, 5(4).

Fumarola, L.; Spinelli, R.; Brandonisio, O. 2004. In vitro assays for evaluation of drug activity against Leishmania spp. Res in Microbiol, 155 (4): 224230.

Fundação de vigilância em Saúde do amazonas FVS-AM, 2012. Indicadores de Morbidade - AM. Disponível em: (http://www.fvs.am.gov.br/) Acesso em 10 Dez. 2016.

Godinho, L.S.; Carvalho, L.S.A.; Castro, C.C.B.; Dias, M.M.; Pinto, P.F.; Crotti, A.E.M.; Pinto, P.L.S.; Moraes, J.; Filho, A.A.S. 2014. Anthelmintic activity of crude and essential oil of Tanacetum vulgare (Asteraceae) against adult worms of Schistosoma mansoni. The Scientific World Journal, ID 460342: 1-9.

Guerreiro, K.K.; Bobek, V.; Santos, V.L.P.; Franco, C.R.C.; Paula, J.P.; Farago, P.V.; Budel, J.M. 2016. Análise farmacobotânica de folha e caule de Tanacetum vulgare (L.). Revista Brasileira de Plantas Medicinais, 18
(1): 89-95.

Jaffe, C.L.; Grimaldi, G.; Pinero, D. 1984. The cultivation and cloning of Leishmania. A Laboratory Manual. 2ed. Fundação Oswaldo Cruz. 91p.

Jansen, F.H. 2006. The herbal tea approach for artemesinin as a therapy for malaria. Transactions of the Royal Society of Tropical Medicine and Hygienev, 100(3): 285-286.

Lahlou, S.; Tahraoui, A.; Israili, Z.; Lyoussi, B. 2007. Diuretic activy of the aqueous extracts of Carum carvi and Tanacetum vulgarein normal rats. Journal of Ethnopharmacology, 110(3): 458-463.

Lorenzi, H.; Matos, F. J. A. 2002. Plantas Medicinais no Brasil: Nativas e Exóticas. Nova Odessa: Instituto Plantarum. 544p.

Luize, P.S.; Tiuman, T.S.; Morello, L.G.; Maza, P.K.; Ueda-Nakamura, T.; Filho, B.P.D.; Cortez, D.A.C.; Mello, J.C.P.; Nakamura, C.V. 2005. Effects of medicinal plant extracts on growth of Leishmania $(L$. amazonensis and Trypanosoma cruzi. Revista Brasileira de Ciências Farmacêuticas, 41(1): 85-94.

Mota, L.A.A.; Miranda, R.R. 2011. Manifestações dermatológicas e otorrinolaringológicas na Leishmaniose. Arquivos Internacionais de Otorrinolaringologia, 15(3): 376-381.

Schinella, G.R.; Giner, R.M.; Recio, M.D.C.; Buschiazzo, P.M.; Ríos, J.L.; Mánez, S. 1998. Anti-inflamatory Effects of South American Tanacetum vulgare. Pharmacy and Pharmacology, 50(9): 1069-1074.

Schinor, E.C.; Salvador, M.J.; Pral, E.M.F.; Alferi, S.C.; Albuquerque, S.; Dias, D.A. 2007. Effect of extracts and isolated compounds from Chresta scapigera on viability of Leishmania amazonensis e Trypanosoma cruzi. Revista Brasileira de Ciências Farmacêuticas, 43(2): 295-300.

Silva, B.P.; Cortez, D.A.; Violin, T.Y.; Filho, B.P.D.; Nakamura, C.V.; UedaNakamura, T.; Ferreira, I.C.P. 2010. Antileishmanial activity of a guaianolide from Tanacetum parthenium (L.) Schultz Bip. Parasitology International, 59 (4): 643-646.

Silva-Filho, A.A.; Resende, D.O.; Fukui, M.J.; Santos, F.F.; Pauletti, P.M.; Cunha, W.R.; Silva, M.L.A.; Gregório, L.E.; Bastos, J.K.; Nanayakkara, N.P.D. 2009. In vitro antileishmania, antiplasmodial and cytotoxic activities of phenolics and triterpenoids from Baccharis dracunculifolia D. C. (Asteraceae). Fitoterapia, 80(8): 478-482.

Silva-López, R.E. 2010. Proteases de Leishmania: novos alvos para o desenvolvimento racional de fármacos. Química Nova, 33(7): 15411548 .

Souza, C.C.V. 2010. Etnobotânica de quintais em três comunidades ribeirinhas na Amazônia Central, Manaus-AM. Dissertação de Mestrado em Botânica, Instituto Nacional de Pesquisas da Amazônia, Manaus, Amazonas. 103p.

Sistema de Informação de Agravos de Notificação-SINAN. Sistema de Informação de Agravos de Notificação. Leishmaniose Tegumentar. Acesso em: 04 Fevereiro 2017.

Pinheiro, F.G.; Luz, S.L.B.; Franco, A.M.R. 2008. Infecção natural por tripanosomatídeos (Kinetoplastida: Trypanosomatidae) em Lutzomyia umbratilis (Diptera: Psychodidae) em áreas de leishmaniose tegumentar americana no Amazonas. Acta Amazônica, 38 (1): 165-172.

Rabito, M.F.; Britta, E.A.; Pelegrini, B.L.; Scariot, D.B.; Almeida, M.B.; Nixdorf, S.L.; Nakamura, C.V.; Ferreira, I.C.P. 2014. In vitro and in vivo antileishmania activity of sesquiterpene lactone-rich dichloromethane fraction obtained from Tanacetum parthenium (L.) Schultz-Bip. Experimental Parasitology, 143: 18-23

Pessini, G.L.; Holetz, F.B.; Sanches, N.R.; Cortez, D.A.G.; Dias Filho, B.P.; Nakamura, C.V. 2003. Avaliação da atividade antibacteriana e antifúngica de extratos de plantas utilizados na medicina popular. Revista Brasileira de Farmacognosia, 13(supl. 1): 21-24.

Pigott, D.M.; Golding, N.; Messina, J.P.; Battle, K.E.; Duda, K.A.; Balard, Y.; Bastien, P.; Pratlong, F.; Brownstein, J.S.; Freifeld, C.C.; Mekaru, S.R.; Madoff, L.C.; George, D.B.; Myers, M.F.; Hay, S.I. 2014. Global database of leishmaniasis occurrence locations, 1960-2012. Scientific Data, 1: 1-7.

Osorio, E.; Arango, G.J.; Jiménez, N.; Alzate, F.; Ruiz, G.; Gutiérrez, D.; Paco, M.A.; Giménez, A.; Robledo, S. 2007. Antiprotozoal and cytotoxic activities in vitro of Colombian Annonaceae. Journal of Ethnopharmacology, 111(3): 630-635.

WHO. 2010. Control of the leishmaniases. World Health Organ Tech Rep Ser. 949: 1-186. ISSN 0512-3054.

Willians, C. A.; Harbone, J.B.; Geiger, H.; Hoult, J.R.S. 1999. The flavonoids of Tanacetum parthenium and Tanacetum vulgare and their antiinflamatory properties. Phytochemistry, 51(3): 417-423. 